\title{
La myopathie de Duchenne du gène DMD à la dystrophine
}

Le gène de la myopathie de Duchenne est un «phénomène »: il est le plus grand de tous les gènes connus à ce jour. La stratégie ayant conduit à sa reconnaissance est exemplaire de la démarche actuelle de la génétique et en représente l'un des plus éclatants succès. Les mutations responsables de la maladie sont, dans plus de la moitié des cas, des délétions géniques et constituent souvent des événements récents posant de redoutables problèmes pour le conseil génétique. La protéine pour laquelle code le gène a été dénommée «dystrophine». Son poids moléculaire est de 40000 et elle serait associée à la «triade», structure intervenant dans les mouvements de calcium dans le muscle.

\section{Jamel Chelly \\ Docteur en médecine}

Jean-Claude Kaplan Professeur au CHU Cochin

\section{ADRESSE}

J. Chelly, J.-C. Kaplan: Institut de pathologie moléculaire, Inserm U. 129, CHU Cochin, 24, rue du Faubourg-Saint-Jacques, 75014 Paris, France.

$m / s n^{\circ} 2$ vol. 4, mars 88 a myopathie de Duchenne ou dystrophie musculaire de Duchenne (DMD) est une maladie dégénérative de la fibre musculaire, individualisée par Duchenne en 1860.

Son tableau clinique est marqué par l'apparition de troubles de la marche vers trois ans et une atrophie musculaire progressive conduisant les enfants au fauteuil roulant vers l'âge de dix ans et les adolescents vers l'insuffisance respiratoire et la mort vers l'âge de 20 ans. Elle touche un garçon sur 3500 .

Jusqu'à ce jour, aucun traitement ne s'est montré capable d'enrayer le cours inexorable de la maladie. Seules des mesures palliatives dans le domaine de l'orthopédie et de la réanimation permettent de prolonger la vie au-delà de la $20^{\mathrm{e}}$ année. A côté de cette forme très sévère, il existe une forme plus tardive et évoluant beaucoup plus lentement, individualisée par Becker en 1955. La myopathie de Becker (BMD) n'est totalement invalidante que plus tard dans la vie adulte, permettant aux sujets qui en sont atteints de procréer et d'avoir une vie socio-professionnelle. Elle est dix fois moins fréquente que la myopathie de type Duchenne.

A l'aube des années 1980, notre savoir sur les maladies de Duchenne et de Becker se limitait à une bonne connaissance de la symptomatologie clinique et à la notion d'une hérédité récessive liée au sexe : seuls les garçons sont atteints, les mères transmettrices sont asymptomatiques mais ont dans deux tiers des cas une élévation de la créatine kinase plasmatique.

Malgré des recherches intensives, aucun mécanisme physiopathologique n'avait été démontré et aucun indice ne permettait de mettre en cause une protéine musculaire particulière.

Dès leur apparition les techni- 


\section{RÉFÉRENCES}

1. Williamson R. Antenatal diagnosis of genetic defects. Nature 1978 ; 276 : 114-5.

2. Botstein D, White RL, Skolnick M, Davis RK. Construction of a genetic linkage map using restriction fragment length polyporphisms Am J Hum Genet 1980; 32 : 31431 .

3. Kunkel LM, Monaco AP, Middlesworth W, Ochs HD, Latt SA. Specific cloning of DNA fragments absent from the DNA of a male patient with an $\mathrm{X}$ chromosome deletion. Proc Natl Acad Sci USA 1985 ; 82 : 4778-82.

4. WortonRG, DuffC, SylvertonJE, Schmickel RD, Willard HF. Duchenne muscular dystrophy involving translocation of the gene next to ribosomal RNA genes. Science 1984 ; 224 : 1447-9.

5. Monaco AP, Bertelson CJ, Middlesworth $W$, et al. Detection of deletions spanning the Duchenne muscular dystrophy locus using a tighthy linked DNA fragment. Nature $1985 ; 316: 842-5$

6. Kunkel LM, Hejtmoncile JF, Caskey CT et al. Analysis of deletions in the DNA of patients with Becker and Duchenne muscular dystrophy. Nature 1986 ; 322 : 73-7.

7. Greenstein RM, Reardon MP, Chan TS. An $\mathrm{X}$ /autosome translocation in a girl with Duchenne muscular dystrophy (DMD) : evidence for DMD gene localisation. Pediatr Res 1977 ; $11: 475 \mathrm{~A}$.

8. Verellen-Dumoulin C, FreundM, Meyer R. Expression of an X-linked muscular dystrophy in a female due to translocation involving $\mathrm{Xp} 21$ and non randon inactivation of the normal X chromosome. Hum Genet $1984 ; 67$ : 115-9.

9. Boyd Y, Buckle VJ. Cytogenetic heterogeneity of translocations associated with Duchenne muscular dystrophy. Clin Genet ques de génie génétique ont laissé entrevoir la possibilité d'une démarche entièrement nouvelle consistant à localiser sur le génome, puis à cloner et en fin de compte à identifier les gènes inconnus qui, comme pour DMD et BMD, se manifestent par une pathologie génétique indiscutablement monofactorielle [1, 2].

Cette approche consistant à isoler d'abord un gène inconnu, puis à en déduire son contenu informationnel et la protéine qu'il spécifie, a reçu le nom mérité de «génétique inverse».

Commencée en 1980, la quête du gène DMD a fini par conduire au but en 1986-1987 [3-6, 31, 33].

\section{De la maladie au locus}

Localisation du locus DMD/ BMD en Xp21. (a). L'approche cytogénétique. Si l'on savait depuis longtemps que la dystro- phie musculaire de Duchenne est une maladie récessive liée au chromosome $\mathrm{X}$, la suspicion d'une localisation du locus DMD sur le bras court de ce chromosome, et plus particulièrement dans la bande Xp2l remonte à 1977 [7, 8]. En effet l'attention a été attirée sur des cas de DMD survenant chez des filles portant des translocations ( $X$; autosome), où le point de cassure est variable sur les autosomes mais constant, en Xp21, sur le chromosome X (figure 1).

L'exploration cytogénétique fine de cette anomalie a permis de suggérer que la conséquence phénotypique (maladie de Duchenne) est due à la combinaison suivante: (a) désorganisation de la structure du locus DMD due à la cassure chromosomique en Xp2l ; (b) l'inactivation préférentielle du chromosome $\mathrm{X}$ intact*.

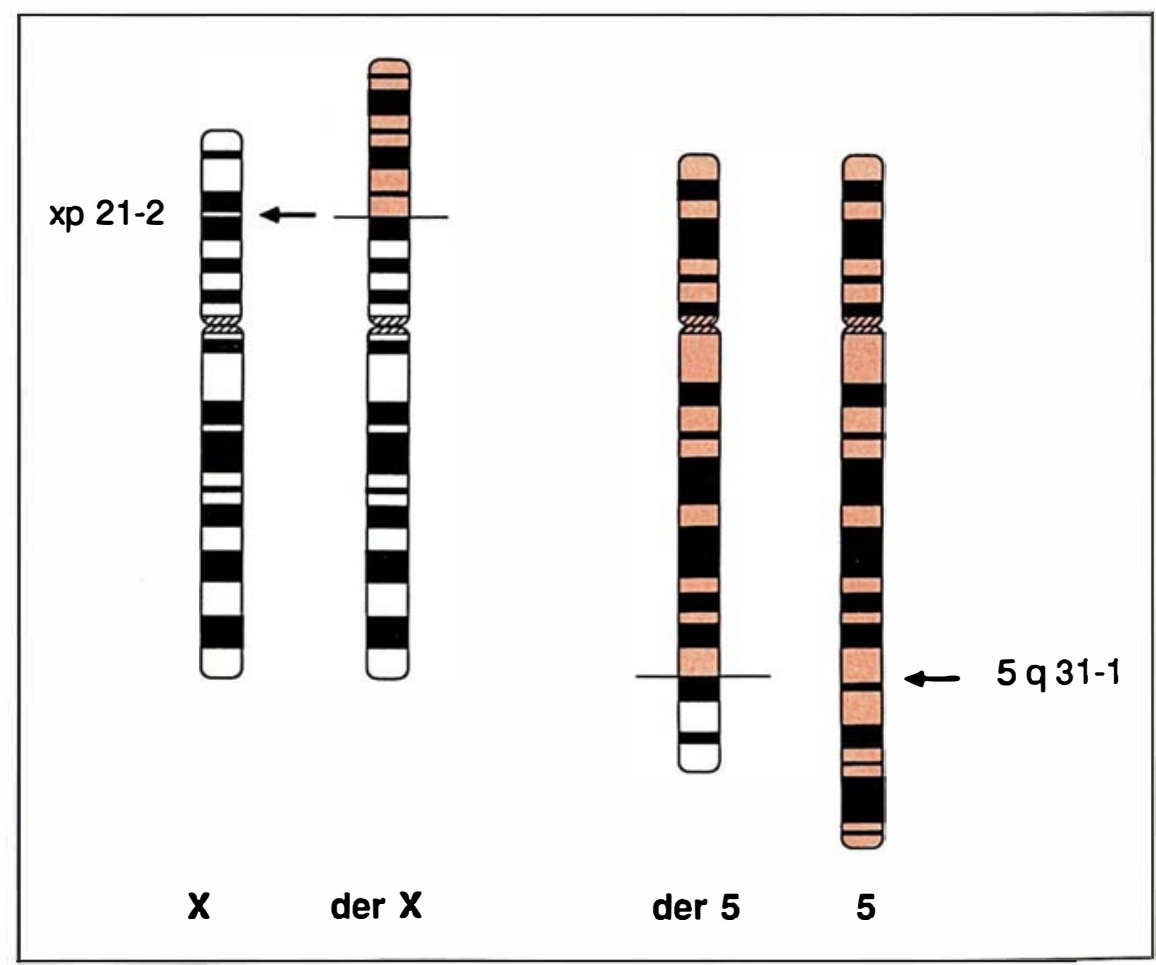

Figure 1. Exemple de translocation ( $X$; autosome) observée chez une fille atteinte de DMD. $t(X ; 5)(p 21.2 ; q 31.1)$. Le chromosome $X$ est composé de bandes noires et blanches, l'autosome (chromosome 5) est composé de bandes noires et rouges. der $X$ et der $5=$ chromosomes $X$ et 5 remaniés après la translocation. 


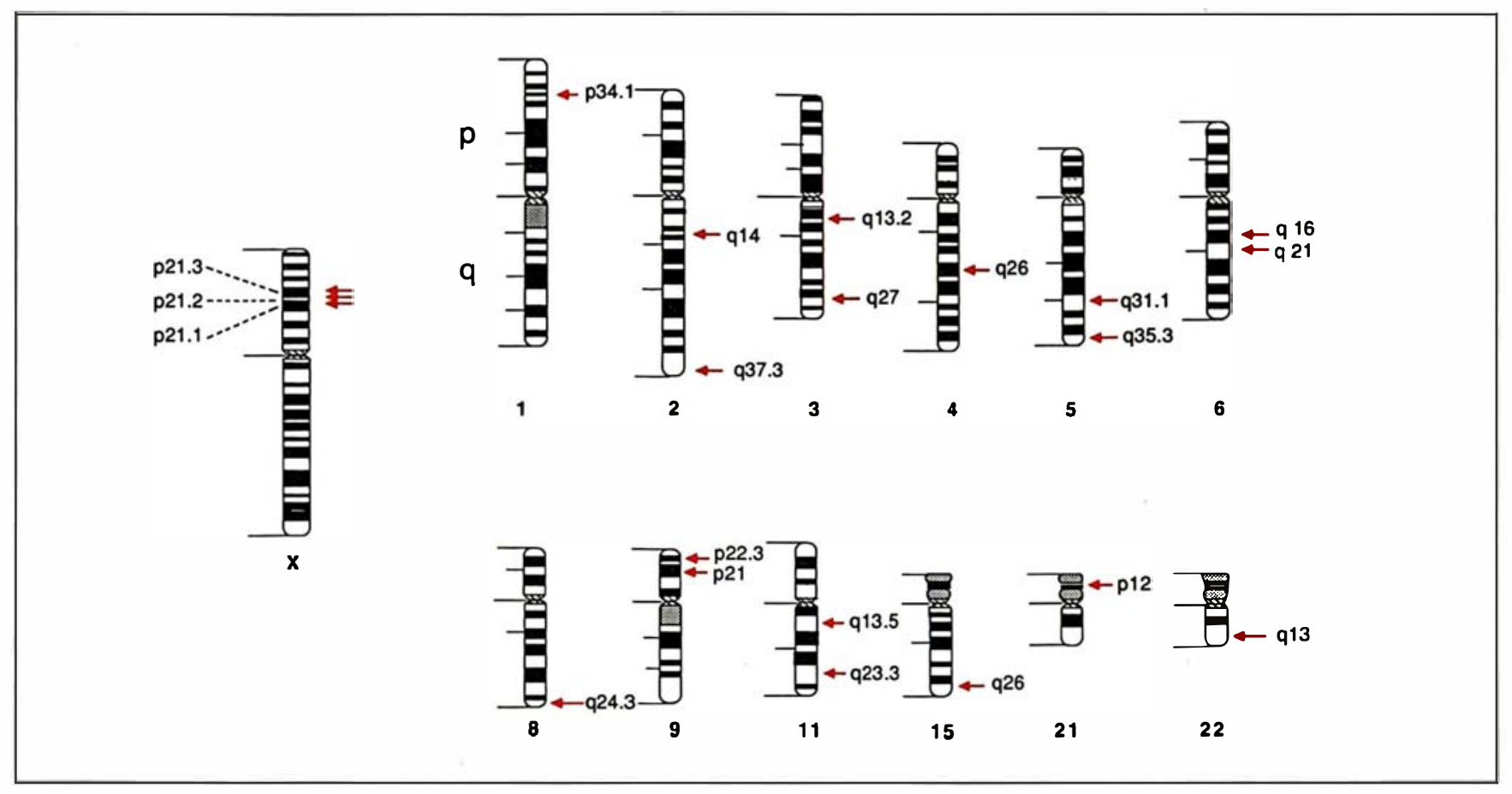

Figure 2. Illustration des 18 cas de translocations ( $X$; autosomes) observées chez des filles atteintes de dystrophie musculaire de Duchenne. Les flèches rouges indiquent les points de cassure. Au niveau du chromosome $X$ la cassure survient toujours dans la bande Xp21 avec une hétérogénéité locale (Xp21.1, Xp21.2, Xp21.3), alors que le point de cassure au niveau des autosomes est variable.

On connaît actuellement 18 cas de translocation (X; autosome) chez des filles atteintes de DMD. Si le chromosome $\mathrm{X}$ est toujours cassé en Xp21, l'analyse cytogénétique en pro-métaphase révèle une micro-hétérogénéité des points de cassure au niveau de la bande Xp2l (figure 2) [9]. La variabilité du point de cassure dans la bande Xp2l a été confirmée récemment par une cartographie moléculaire des translocations, utilisant une série de sondes localisée en Xp2l et par hybridation in situ appliquée à des hybrides somatiques ayant retenu le chromosome transloqué $[9,10]$.

Ces données cytogénétiques évoquaient déjà 2 hypothèses : ou bien le gène responsable de la maladie est très étendu; ou bien son expression peut être affectée par des événements se produisant à distance.

La deuxième anomalie cytogénétique confortant les soupçons portant sur la région Xp21, est la constatation de microdélétions de la bande Xp21, chez des garçons atteints d'une association morbide: DMD associée à une ou plusieurs des entités suivantes: granulomatose chronique; rétinite pigmentaire; déficit en glycérol kinase; hypoplasie surrénale congénitale [11, 12] (figures 3 et 4, page 144).

(b). Les analyses de linkage avec les premières sondes indirectes (extragéniques). Le bien-fondé de la localisation suggérée par la cytogénétique a été confirmé par des analyses génétiques de linkage, utilisant des marqueurs d'ADN de cette région détectant des polymorphismes de restriction (RFLP : restriction fragment length polymorphism ${ }^{* *}$. Les premiers

\footnotetext{
* En effet, en cas de translocations (X; autosome) équilibrées il est de règle que le chromosome X normal soit préférentiellement inactivé.

**Voir m/s, suppl. au no 7, vol.3, p. 4.
}

RFLP identifiés ont été révélés par les sondes RC8 (locus DXS9)[13] et L1.28 (locus DXS7) [14]. Des recombinaisons ont été trouvées, permettant de localiser ces locus de part et d'autre du gène DMD à une distance d'environ 17 centimorgans. Ces sondes étaient donc encore trop éloignées pour être utilisées à des fins diagnostiques. Mais elles fournissaient déjà deux renseignements précieux: (a) le domaine recherché était cerné dans la région suspectée au départ; (b) le locus de la myopathie de Becker (BMD) donnait les mêmes résultats à l'analyse de linkage, ce qui suggérait son identité avec le locus DMD [15].

Pour progresser il fallait trouver de nouvelles sondes qui soient à la fois plus rapprochées, pour minimiser les risques de recombinaison, et aussi polymorphes que possible, pour augmenter l'informativité. Ainsi une panoplie de sondes réparties de part et d'autre 


\section{RÉFÉRENCES}

10. Boyd Y, Buckle V, Holt S, Munro E, Hunter D, Craig AI. Muscular dystrophy in girls with X; autosome translocations. J Med Genet 1986 ; 23 : 484-90.

11. Francke U, Ochs HD, de Martinville B, et al. Minor Xp2l chromosome deletion in a male associated with expression of Duchenne muscular dystrophy, Chronic granulomatous disease, retinitis pigmentosa and McLeod syndrome. Am J Hum Genet 1985; 37 : 250 67.

12. Clarke A, Roberts $\mathrm{H}$, Thomas NST, Whitfield A, Williams J, HarperPS. Duchenne muscular dystrophy with adrenal insufficiency and glycerol kinase deficiency: high resolution cytogenetic analysis with molecular, biochemical, and clinical studies. J Med Genet 1986; 23 : 501-8.

13. Murray JM, Davies KE, Harper PS, Williamson $\mathbf{R}$. Linkage relationship of a cloned DNA sequence on the short arm of the $\mathrm{X}$ chromosome to Duchenne muscular dystrophy. Nature $1982 ; 300$ : 69-71.

14. Davies KE, Pearson PL, Harper PS, et al. Linkage analysis of two cloned DNA sequences flanking the Duchenne muscular dystrophy locus on the short arm of the human X chromosome. Nucleic Acids Res. 1983 ; $11: 2303-12$.

15. Kingston HM, Sarfarazi M, Thomas NST, Harper PS. Localisation of the Becker muscular dystrophy gene to the short arm of the $\mathrm{X}$ chromosome by linkage to cloned DNA sequences. Hum Genet 1984; $67: 6-17$.

16. Bakker E, Hofker MH, Goor N, et al. Prenatal diagnosis and carrier detection of Duchenne muscular dystrophy with closely linked RFLPs. Lancet 1985 ; ii : 655-58.

17. Ray PN, Belfall B, Duff C, et al. Cloning of the breakpoint of an $\mathrm{X} ; 2 \mathrm{l}$ translocation associated with Duchenne muscular dystrophy. Nature 1985 ; 318 : 672-5.

18. Monaco AM, Bertelson CJ, Colletti-Feener C, Kunkel LM. Localisation and cloning of Xp2l deletion breakpoints involved in muscular dystrophy. Hum Genet 1987; 75 :

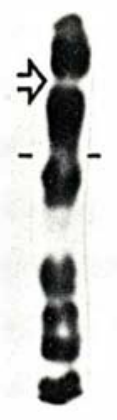

A

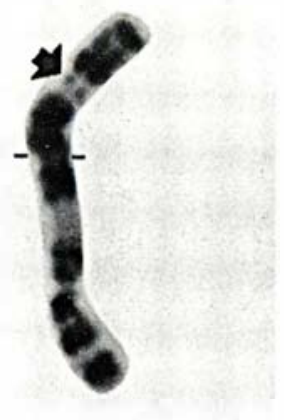

B
Figure 3. Exemple de délétion cytogénétique observée chez un garcon atteint d'une association morbide: DMD-déficit en glycérol kinase, hypoplasie surrénale congénitale et arriération mentale [26]. (A). chromosome $X$ du propositus: microdélétion de la bande $X p 21.2(\Leftrightarrow)$. (B). chromosome $X$ normal d'un garçon normal $\rightarrow$ ). (Photo: laboratoire B. Dutrillaux.)

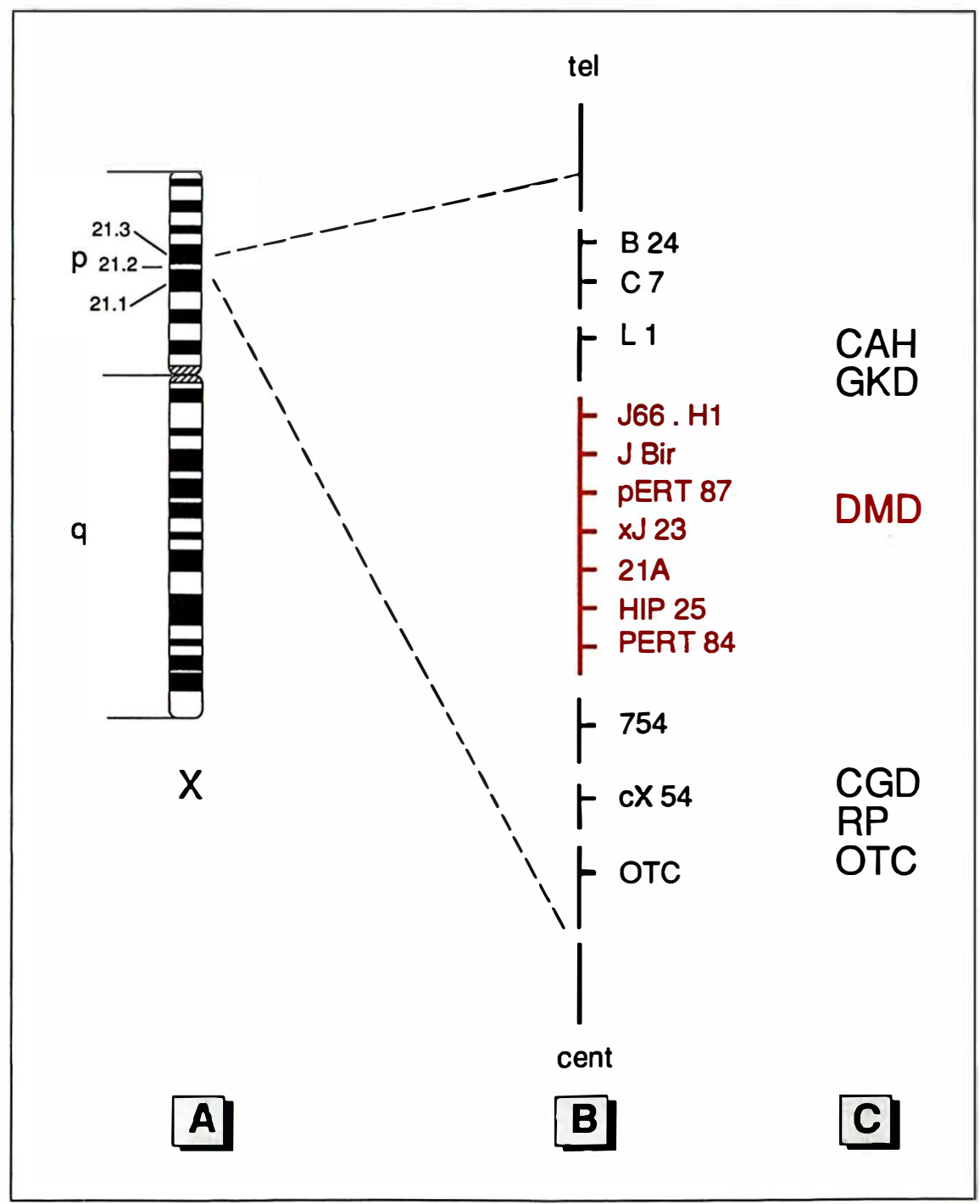

Figure 4. Localisation ordonnée des différents marqueurs du DNA, et carte morbide de la bande Xp21. (A). Idéogramme en bande G du chromosome $X$. (B). Localisation et ordre des différentes sondes de la bande Xp21, la discontinuité indique que la distance entre deux marqueurs successifs n'est pas connue. (C). Carte morbide de la région Xp21. CAH = congenital adrenal hypoplasia (hypoplasie congénitale des surrénales); GKD = glycerol kinase deficiency (déficit en glycérol kinase) ; $C G D=$ chronic granulomatous disease (= GC, granulomatose chronique ; $R P=$ rétinite pigmentaire $; O T C=$ ornithine transcarbamylase). 


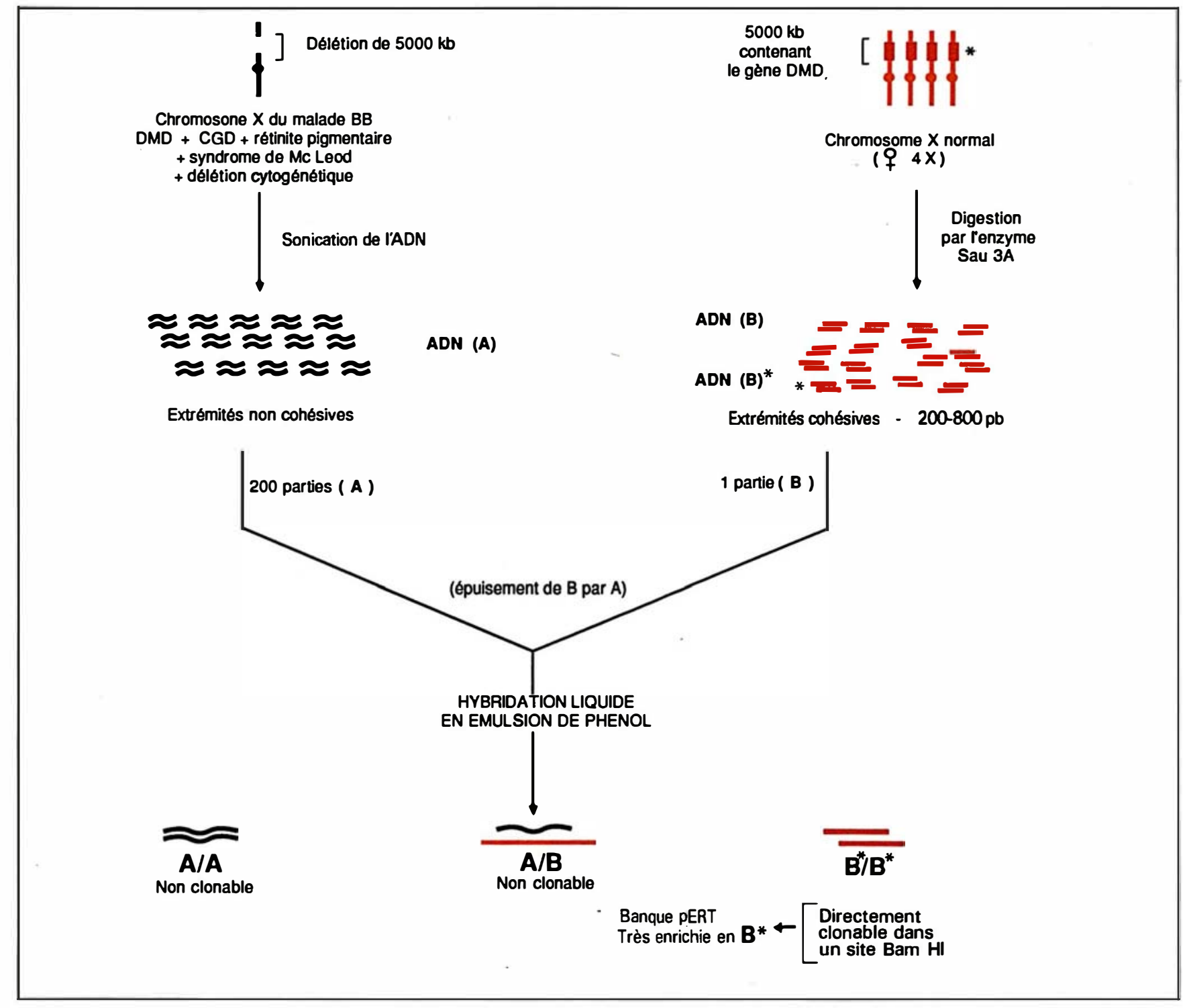

Figure 5. Stratégie de clonage direct d'un fragment d'ADN correspondant à une délétion cytogénétique (stratégie de L. Kunkel appliquée au gène DMD). La technique pERT (phenol enhanced reassociation technique), c'està-dire la vitesse de réassociation des brins complémentaires, multiplie le Cot par 20000. La réassociation complète des séquences uniques est obtenue en une semaine au lieu de 20000 semaines (384 ans). La banque pERT est considérablement enrichie en séquences contenant notamment des fragments de gène DMD et de gène CGD (chronic granulomatous disease).

du locus DMD a été isolée, permettant d'effectuer les premiers diagnostics prénatals avant même que le gène ait été isolé [16].

\section{Du locus au gène}

Les premières sondes incragéniques du locus DMD/BMD. La première stratégie a consisté à cloner le fragment de jonction de la translocation $(\mathrm{X} ; 2 \mathrm{2l})$ au niveau d'un point de cassure autosomique localisé dans le gène $28 \mathrm{~S}$ $m / s n^{\circ} 3$ vol. 4 , mars 88 ribosomal du chromosome 21. Le clone de jonction (XJ) contenait des fragments d'ADN spécifiques de la région $\mathrm{Xp} 2 \mathrm{l}$ très liés $\mathrm{au}$ locus DMD, et identifiant des anomalies moléculaires chez des patients atteints uniquement de DMD $[4,17]$.

La deuxième stratégie utilisée a consisté à explorer une délétion cytogénétique interstitielle en Xp2l observée chez un garçon atteint de l'association DMD + GC (granulomatose chronique ou CGD, chronic granulomatous disease) + RP rétinite pigmentaire [11]. L'ADN de ce malade a été utilisé en large excès dans une expérience d'hybridation compétitive avec de l'ADN normal en émulsion phénolique (pour accélérer la cinétique de réassociation). La saturation des séquences homologues d'ADN normal par un excès d'ADN du malade a permis un enrichissement en séquences absentes dans le génome du malade [3] (figure 5). 


\section{RÉFÉRENCES}

19. Van Ommen CJB, Bertelson C, Cinjaar $\mathrm{HB}$, et al. Long range genomic map of the Duchenne muscular dystrophy gene; isolation and use of J66 (DXS 268), as distal intragenic marker. Genomics (sous presse).

20. Monaco AP, Neve R, Colletti-Feener C, Bertelson CJ, Kurnit DM, Kunkel LM. Isolation of candidate cDNAs for portions of the Duchenne muscular dystrophy gene. Nature 1986 ; 323 : 646-50.

21. Burmeister $\mathbf{M}$, Lehrach $H$. Long Range restriction map around the Duchenne muscu lar dystrophy gene. Nature 1986 ; 324 : 582-5.

22. Carle GF, Frank M, Olson MV. Electrophoresis separations of large DNA molecules by periodic inversion of the electric field. Science $1986 ; 4: 65-8$.

23. Van Ommen CJB, Verkerk JMH, Hofker $\mathrm{MH}$, et al. A physical map of 4 million bp around the Duchenne muscular dystrophy gene on the human $\mathrm{X}$ chromosome. Cell $1986 ; 47: 499-504$

24. Robertson M. Muscular Dystrophy. Mapping the disease phenotype. Nature 1987 $327: 372-3$

25. Wood DS, Zeviani M, Prelle A, et al. Is nebulin the defective gene product in Duchenne muscular dystrophy. N Engl J Med $1987 ; 316: 107-8$.

26. Pernelle JJ, Chafey P, Chelly J, Wahrmann P, Kaplan JC. Nebulin seen in DMD males including one patient with a large DNA deletion encompassing the DMD gene.
Cette stratégie a permis d'isoler sept fragments d'ADN localisés en Xp2l et absents chez le malade. En utilisant ces fragments comme nouvelles sondes, et en les appliquant à l'analyse de l'ADN de malades atteints de myopathie de Duchenne classique, l'équipe de Kunkel devait constater que seule la sonde pERT 87 détectait des délétions dans une proportion significative de ces cas ( 5 cas sur les 57 premiers cas explorés) [5].

Une marche bidirectionnelle sur le chromosome X à partir de la séquence d'origine pERT 87 (200 pb) a abouti au clonage d'une région de $220 \mathrm{~kb}$ (locus DXS 164) et à l'isolement de plusieurs sondes qui reconnaissent des séquences uniques et des polymorphismes de restriction [5, 6].

Les locus pERT 87 et $X J$ sont dans le gène DMD. La distribution des sondes pERT 87, l, 8 et 15 et XJ1.2 et XJ2.3 à la communauté scientifique a permis une étude collaborative internationale [6]. L'analyse de l'ADN de 1346 malades atteints de DMD ou de BMD a montré : des délétions dans les deux types de maladie; une fréquence des délétions de l'ordre de $7 \%$; une forte liaison des sondes du domaine DXS 164 (pERT 87) et DXS 102 (XJ) au locus DMD; l'existence de $5 \%$ de recombinaisons entre les sondes pERT et les mutations affectant le locus DMD; l'absence de segment délété commun à toutes les délétions, celles-ci dépassant souvent 100 kilobases $(\mathrm{kb})$, dans les deux directions du locus DXS 164 avec et sans recouvrement [6]. Par ailleurs, la détection directe des points de cassure de certaines délétions, révélées par des fragments de taille anormale en Southern blot après hybridation avec les sondes de la région pERT 87 , a permis d'effectuer des sauts et le clonage de quatre locus situés à une distance importante de la région pERT 87 [18, 19].

La cartographie physique des 4 nouveaux locus: J.47 et J.MD proximaux par rapport au clone de jonction $\mathrm{XJ}$ de la translocation (X; 21), J.Bir et J66. $\mathrm{H}_{1}$ distaux par rapport au domaine
pERT 87, ne montre aucun chevauchement avec le locus initial pERT 87 ; de plus, les sondes isolées à partir de ces nouveaux domaines ont également révélé des délétions chez des myopathes (figure 6).

A la recherche d'un transcrit du gène $\mathbf{D M D} / \mathbf{B M D}$. L'hétérogénéité et l'étendue des délétions, ainsi que les données d'analyse de ségrégation indiquent que le locus DMD correspond à un gène très étendu ou bien à plusieurs petits gènes. La stratégie adoptée pour identifier les clones de la région pERT 87 susceptibles de contenir des exons du gène DMD a reposé sur la recherche de séquences très conservées chez plusieurs espèces animales (stratégie dite du Zoo-blot consistant à hybrider une sonde avec de l'ADN génomique provenant d'espèces de plus en plus éloignées: mammifères, oiseaux, poissons, insectes, etc.).

Ainsi la séquence nucléotidique de deux fragments de la région pERT 87 est hautement conservée (mammifère et poulet), possède une structure évocatrice d'exon avec un cadre de lecture ouvert délimité par des sites 5' et 3' d'épissage. L'hybridation de l'un de ces deux clones avec de l'ARN total et de la fraction polyadénylée (enrichie en ARN messagers puisqu'ils sont polyadénylés alors que les ARN ribosomaux ne le sont pas) a permis d'identifier un long transcrit de $16 \mathrm{~kb}$ dans le muscle squelettique (humain et de souris) [20].

Le même fragment génomique a été utilisé pour isoler des clones d'ADN complémentaire du messager $(\mathrm{ADNc})$ correspondant à $10 \%$ du transcrit. L'hybridation des séquences d'ADNc avec de l'ADN génomique a révélé une organisation particulière du gène: les exons correspondant à l'ADNc ont une taille moyenne de $150 \mathrm{pb}$ et seraient séparés par des introns d'environ $16 \mathrm{~kb}$. Comme $1 \mathrm{~kb}$ de cet ADNc hybride avec environ $130 \mathrm{~kb}$ de DNA génomique, une extrapolation de ce résultat partiel permet de déduire que le transcrit de $16 \mathrm{~kb}$ corres- 
pond à au moins 100 fois plus sur le génome, soit 1600 à $2000 \mathrm{~kb}$ [20].

Structure globale du gène DMD/BMD. La taille très importante du gène est un fait sans précédent dans tout le règne animal ou végétal.

Les extrémités 5' et 3' du gène n'ont pas encore été identifiées avec certitude, mais une région riche en CG et hypométhylée (îlot HTF : Hpa II tiny fragment, voir revue générale dans [34]) a été localisée à proximité du locus pERT 84 [21]. Le site d'initiation du gène dans cette région et un ATG initiateur de la séquence codante y ont été identifiés par l'équipe de Kunkel. Quant à l'ex- trémité 3' du gène, elle se trouve entre les marqueurs $\mathrm{J} 66 . \mathrm{H}_{1}$ et $\mathrm{L}_{1}$ (ce résultat est basé sur l'analyse de l'ADN d'un malade atteint de DMD, de déficit en glycérol kinase et d'hypoplasie surrénale congénitale avec délétion de $\mathrm{C}_{7}$ et de $\mathrm{L}_{1}$ sans délétion de $\left.\mathrm{J} 66 . \mathrm{H}_{1}\right)$. Le clonage de l'ADNc a permis l'identification des premiers exons.

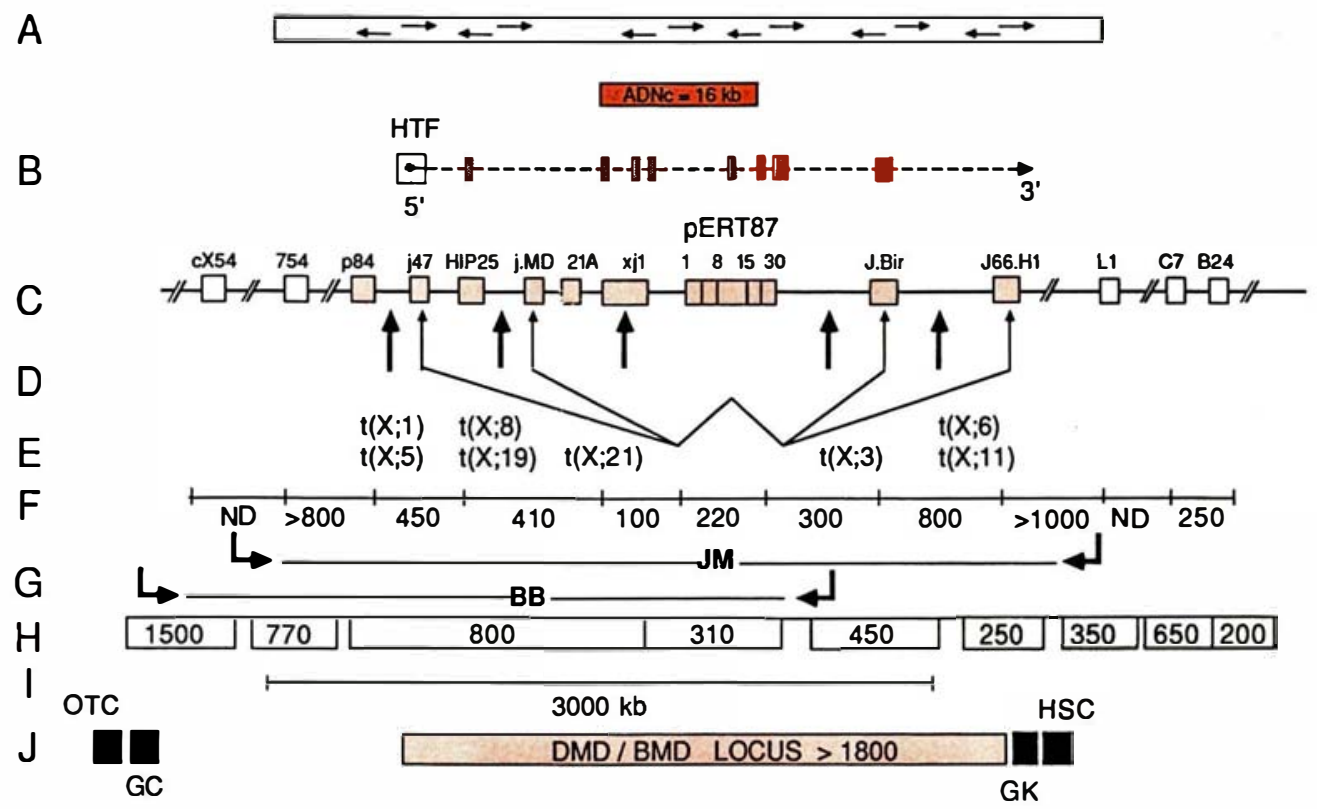

Figure 6. Schéma du locus DMD/BMD et des régions flanquantes. (A). Le rectangle indique la région touchée par les délétions observées dans les différents cas de DMD. Les flèches indiquent : (a) l'orientation centromérique ou télomérique des délétions; (b) l'absence de séquence commune délétée; (c) l'hétérogénéité des points de cassure des délétions. (B). Représentation de quelques séquences (exons) qui ont hybridé avec des clones d'ADNc obtenus à partir du transcrit de $16 \mathrm{~kb}$ (kilobases) repéré dans le muscle squelettique. La boîte HTF (Hpa II tiny fragment), région riche en CGD (chronic granulomatous disease), non méthylés, a été localisée entre les sondes pERT 84 et j.47; les séquences HTF ont été retrouvées dans la région 5' de plusieurs gènes domestiques [34]. L'extrémité 5' du gène DMD serait à proximité de cette région HTF. (C). Position des locus de la région Xp21 et des sondes correspondantes à chaque domaine. (cX54 - DXS48; 754 - DXS84; p84 - DXS142; xj1 - DXS206; pERT87 - DXS164; L1 - DXS68; C7 - DXS28; B24 - DXS67). Pour : j.47; j.MD; HIP 25; xj1 ; J.Bir; J66.H1 : la désignation des locus selon HGM (human gene mapping, conférence de nomenclature des locus sur I'ADN humain) n'est pas encore faite. L'ordre des locus représenté par les sondes L1, C7 et B24 a été établi par l'analyse de délétions chez des patients atteints d'associations morbides. Les blocs pleins indiquent les domaines inclus dans le gène; les blocs-creux indiquent les domaines extra-géniques. (D). Les locus j.47; j.MD; J.Bir; J66.H1 ont été clonés à partir de I'ADN de malades atteints de DMD avec délétions moléculaires (un des deux points de cassure est situé dans le domaine pERT 87) après identification des fragments de jonction avec des sondes isolées du locus pERT 87. (E). Hétérogénéité et répartition des points de cassure. Les flèches noires indiquent les régions remaniées par les points de cassure des translocations $X$; autosomes responsables de phénotype DMD chez les filles porteuses de ces remaniements cytogénétiques. (F). Distances en kilopaires de bases entre les différents locus déduits à partir des macrocartographies publiées par G.J.B. Van Ommen (1986) et M. Burmeister (1986). ND: distance non déterminée. (G). Représentation des délétions identifiées chez: (a). JM $=$ patient atteint de DMD (HSC = déficit en GK) et de retard mental avec délétion d'environ $4000 \mathrm{~kb} ;(\mathrm{b})$. BB = patient atteint de $D M D$ + granulomatose chronique + rétinite pigmentaire. Les flèches noires indiquent les limites des délétions. (H). Ordre et taille des fragments de restrictions Sfil identifiés par électrophorèse en champ pulsé, répartis sur environ $5000 \mathrm{~kb}$ de la bande Xp21. De nouveaux fragments Sfil peuvent exister entre certains locus de la région Xp21, ceci est indiqué par la discontinuité de la carte des locus (C) et des fragments Sfil. (I). Fragment de restriction Not I (3000 kb) séparé par électrophorèse en champ pulsé, ce même fragment hybride avec trois sondes différentes: 754, xj12 et J.Bir. (J). Carte morbide de la région Xp21 et position relative des différents locus par rapport aux différentes sondes de la région. 


\section{REFEERENCES}

27. Bertelson CJ, Bartley JA, Monaco AM, Colletti-Feener C, Fishbeck K, Kunkel LM. Localisation of $\mathrm{Xp} 21$ meiotic exchange points in Duchenne muscular dystrophy families. J Med Genet 1986 ; 23 : 531-7.

28. Koenig M, Hoffman EP, Bertelson CJ, et al. Complete cloning of the Duchenne Muscular Dystrophy (DMD) cDNA and preliminary genomic organization of the DMD gene in normal and affected individuals. Cell 1987 ; $50: 509-17$

29. Darras BT, Zeviani M, Di Mauro $S$ Schon EA, Francke U. The human nebulin maps to chromosome 2: q 13-q 33. Cytogenet Cell Genet (sous presse).

30. Hoffman EP, Monaco AP, Feener CC, Kunkel LM. Conservation of the Duchenne muscular dystrophy gene in mice and humans. Science 1987 ; 238 : $\mathbf{3 4 7 - 5 0 .}$

31. Hoffman EP, Brown RH, Kunkel LM. Dystrophin: the protein product of the Duchenne muscular dystrophy locus. Cell 1987 ; 51 : 919-28.

32. Hammonds RG Jr. Protein sequence of DMD gene is related to actin-binding domain of $\alpha$-actinin. Cell 1987; $51: 1$.

33. Hoffman EP, Knudson CM, Campbell KP, Kunkel LM. Subcellular fractionation of dystrophin to the triads of skeletal muscle. Nature 1987 ; 330 : 754-8.

34. Bird AP. CPG islands as gene markers in the vertebrate nucleus. Trends Genet $1987 ; 3$ : 342-7.

35. Bakker E, Van Broeckhoven $\mathrm{Ch}$, Bonten EJ. Germline mosaïcism and Duchenne muscular dystrophy mutations. Nature 1987 ; 329 : 554-6.

36. Darras BT, Francke V. A partial deletion of the muscular dystrophy gene transmitted twice by an unaffected male. Nature 1987 ;
l'ADNc a permis l'identification des premiers exons. Leur taille est très réduite : 80 à $120 \mathrm{pb}$ et leur nombre est estimé à environ 70 .

Par ailleurs, la comparaison du gène DMD humain avec celui de la souris a montré une conservation de la taille, de l'organisation et une forte homologie des séquences nucléotidiques à la fois des exons et des introns [24].

L'analyse par ordinateur des parties codantes séquencées $(2,4 \mathrm{~kb}) \mathrm{a}$ montré une structure filamenteuse qui rappelle celles de la myosine et de la spectrine avec des séquences hydrophobes compatibles avec des domaines transmembranaires (résultats présentés au colloque international sur la biologie moléculaire de la myopathie de Duchenne, Versailles, avril 1987) [24].

L'expression du gène a été étudiée dans plusieurs tissus et un ARN messager de $14 \mathrm{~kb}$ a été détecté dans (a) les muscles squelettiques adultes et fotaux; (b) le muscle cardiaque; (c) les myotubes alors que dans le myoblaste la présence du messager est incertaine.

Aucune expression n'a été mise en évidence dans les fibroblastes [24]. Carte à grande échelle de la région Xp21 (figure 6). L'utilisation de l'électrophorèse en champ pulsé après restriction de l'ADN par des enzymes coupant très rarement dans le génome a permis de dresser une carte de restriction couvrant au moins quatre mégabases de la région $\mathrm{Xp} 21$ (avec des lacunes dans certaines régions) [21-23]. L'application de cette technique nouvelle aux anomalies affectant le locus DMD a permis de faire les constatations suivantes: la distance entre les points de cassure des translocations $(\mathrm{X} ; 6)$ et $(\mathrm{X} ; \mathrm{ll})$ dépasse largement $1000 \mathrm{~kb}$; la taille de certaines délétions responsable du phénotype DMD est extrêmement variable ; la distance entre l'extrémité 3 ' du domaine pERT 87 et J.Bir est environ de $300 \mathrm{~kb}$; la distance entre le locus J.Bir et $\mathrm{J} 66 . \mathrm{H}_{1}$ est de $800 \mathrm{~kb}$. La carte de la région obtenue avec les sondes intragéniques permet de prédire que l'ADN génomique total correspondant au locus
DMD couvre un domaine de l'ordre de $2000 \mathrm{~kb}$, car les sondes 754 $\mathrm{XJ}$ l.l et J.Bir hybrident avec un même fragment de restriction Not I de $3000 \mathrm{~kb}$.

Pathologie moléculaire du gène DMD/BMD. Le phénotype DMD, comme le phénotype BMD, est souvent dû à des délétions totales ou partielles du gène DMD, dont la fréquence a d'abord été estimée à $6 \%$ puis à $12 \%$. Mais ce chiffre est certainement sous-estimé car les sondes utilisées n'explorent qu'une partie limitée du gène DMD. La taille et la répartition des délétions sont très hétérogènes (la taille varie de $6 \mathrm{~kb}$ à plus de $2000 \mathrm{~kb}$ et tous les domaines clonés dans ce gène peuvent être affectés par les délétions).

L'étude clinique des différentes formes de DMD n'a pas révélé de corrélation avec les délétions, mais l'étude moléculaire de certaines délétions a permis d'élucider partiellement le déterminisme moléculaire de la différence phénotypique entre DMD et BMD. En effet, Monaco a montré que dans deux cas de DMD avec délétion de 43 et de $180 \mathrm{~kb}$ les points de cassures sont localisés dans des exons et entraînent une perte du cadre de lecture, alors que dans un cas de BMD avec délétion de $6 \mathrm{~kb}$, un exon est perdu mais avec conservation du cadre de lecture et formation très probable d'un produit final dont la fonction n'est pas totalement abolie [24].

La distinction nosologique entre maladies de Beckeret de Duchenne, assez imprécise cliniquement, reçoit ainsi un éclairage moléculaire. La gravité variable est très probablement liée à la nature des exons affectés et au retentissement des mutations sur la transcription et la maturation du messager.

Origine et mécanisme des délétions. Les études familiales des cas de DMD avec délétions à la recherche de l'origine de l'événement délétionnel ont révélé que celui-ci peut se produire aussi bien dans les gamètes maternels que dans les gamètes paternels. L'observation de plusieurs cas de DMD avec duplication du locus 
pERT 87 ou XJ1.1 [27] suggère que le.mécanisme pourvoyeur de délétions pourrait être un crossing over inégal entre chromatides homologues, se produisant au cours de la première division méíotique de la gamétogenèse femelle. En ce qui concerne les gamètes paternels, dans la formation desquels n'intervient qu'un seul chromosome X, les mutations délétionnelles impliquent d'autres mécanismes. Il pourrait s'agir d'échanges intrachromosomiques ou interchromosomiques non homologues.

Par ailleurs l'analyse d'une famille a permis de détecter une délétion étendue $(>1000 \mathrm{~kb})$ chez deux enfants d'une même fratrie (un garçon atteint de DMD et sa sœur) alors que l'étude de l'ADN de la mère n'a révélé aucune anomalie (Chelly et al., résultats non publiés). Au moins 12 cas équivalents à cette observation ont été rapportés au workshop de Versailles 1987 [24] dont deux viennent d'être publiés [35, 36]. La notion de délétion dans une fratrie sans anomalie constitutionnelle du DNA maternel permet d'évoquer un événement mitotique se produisant au stade de l'embryogenèse ou au cours de divisions mitotiques des cellules gamétiques souches, et qui serait à l'origine d'une mosaïque, généralisée ou localisée aux cellules gamétiques.

L'existence de familles avec transmission d'une délétion à plusieurs enfants par une mère qui n'est pas apparemment porteuse de la délétion complique sérieusement le diagnostic anté-natal et le conseil génétique inhérent à cette maladie.

Le conseil génétique' et le diagnostic anténatal. L'énormité du gène (environ $2000 \mathrm{~kb}$ soit 1000 fois plus que le gène de la $\beta$ globine) pose des problèmes nouveaux. La fréquence des formes délétionnelles paraît exceptionnellement élevée (peut-être jusqu'à $50 \%$ ), mais la variété du siège des lésions impose de multiplier les explorations par les sondes génomiques disponibles. On peut espérer une simplifica- tion maintenant qu'il est possible d'utiliser des sondes exoniques (ADNc).

Quant aux mutations apparemment non délétionnelles il semble qu'elles puissent siéger sur n'importe quel point du gène : il en résulte que même l'emploi du RFLP intragénique comporte un risque d'erreur par recombinaison.

Ceci impose l'utilisation de sondes extragéniques encadrant le locus DMD, et un calcul de risque global prenant en compte les différents risques spécifiques (calcul bayésien du risque génétique a priori, créatine kinase, ADN).

Une dernière complication, très préoccupante, provient des observations mentionnées plus haut où une mosaïque maternelle, ou même paternelle, généralisée ou localisée aux cellules germinales, est fortement suspectée. Celles-ci concernaient des formes avec délétion pour les sondes connues, mais on peut craindre que les mosaïques soient aussi présentes en l'absence de délétion. Si tel était le cas, le diagnostic de femme supposée non transmettrice (à cause d'une néo-mutation) comporterait un risque d'erreur impossible à chiffrer dans l'état actuel de nos connaissances.

\section{Du gène à la protéine}

L'ARN messager. Les clones d'ADNc ont permis de caractériser un ARN messager de très grande taille, environ 14 kilobases [20, 28]. Celui-ci a été trouvé jusqu'à présent dans le muscle squelettique adulte et foetal, ainsi que dans le muscle cardiaque [20,28]. Sa taille inusitée et surtout sa très faible abondance, $1 / 10000$ à $1 / 100000$, ont jusqu'à présent empêché d'établir avec précision sa distribution tissulaire, et son expression au cours de la différenciation myoblastique.

Du gène DMD à la dystrophine. Un si long messager correspond vraisemblablement à une protéine de grande taille, de l'ordre de 500 kilodaltons. Parmi les protéines myofibrillaires déjà connues, la nébuline, 500 kilodaltons, corres- pondait à ce signalement, et était absente ou très diminuée dans les muscles de myopathes [25]. Ce n'est pourtant pas le produit du gène $\mathrm{DMD}$, car il peut persister chez des myopathes où la totalité du gène DMD est délétée [26]. D'autre part le gène codant pour la nébuline a pu finalement être localisé sur un autosome, le chromosome 2 [28].

La protéine du gène DMD est en fait une entité originale que l'équipe de L. Kunkel a progressivement reconstituée en déchiffrant la séquence nucléotidique des régions codantes du gène DMD [28-31]. Cette protéine nouvelle, appelée dystrophine [31], a un poids moléculaire de 400 kilodaltons. Elle possède dans sa région $\mathrm{N}$-terminale une structure en hélice $\alpha$ dont la séquence rappelle un domaine polypeptidique déjà rencontré dans l'actinine, protéine se liant à l'actine [32]. Elle est très peu abondante, ne représentant que $1 / 50000$ des protéines musculaires. Sa localisation membranaire, et plus précisément à l'endroit où le reticulum sarcoplasmique entre en contact avec les tubules du système transverse $T$ pour former une triade est une donnée importante dont il faudra tenir compte désormais dans la physio-pathologie de la maladie [33]. Cette découverte redonne vigueur aux thèses, jusqu'ici non démontrées, attribuant un rôle déterminant à des perturbations des échanges calciques intracellulaires, qui entraîneraient une déperdition calcique du reticulum endoplasmique vers le cytosol. Une des principales questions qui se posent à l'heure actuelle est de savoir si le rôle de la dystrophine est structural ou fonctionnel.

De la dystrophine à la mutation mdx de la souris. La souche bien connue de souris atteinte de myopathie héréditaire liée au sexe, dite «mdx», n'était pas jusqu'à présent censée constituer un équivalent murin de la myopathie de Duchenne. Ceci d'une part à cause de son tableau clinique bien différent, caractérisé par un début 
précoce et une évolution régressive de la dystrophie musculaire, qui finalement n'altère pas la longévité de l'animal ; d'autre part, parce que contrairement au modèle humain, le gène DMD de la souris, par ailleurs très semblable au gène humain par sa séquence et son organisation, ne présentait pas de délétion chez les souris mdx. Le locus mdx était donc considéré comme l'équivalent murin du locus de la myopathie humaine moins sévère de type Emery-Dreifuss. Grâce à une étude immunologique, l'équipe de Kunkel a en fait suggéré que les souris de la souche mdx présentent une anomalie primaire de la dystrophine [31]. Si le locus $m d x$ de la souris et le gène de la dystrophine (ex-gène DMD) ne font qu'un, il reste à expliquer pourquoi la maladie évolue si différemment dans les deux espèces. La compréhension de cette différence pourrait orienter les recherches thérapeutiques.

\section{Conclusion}

L'histoire du gène DMD illustre le pouvoir de la stratégie de la génétique inverse.

La découverte récente du gène DMD ouvre un champ d'investigation immense, à la mesure de sa taille. Les connaissances sont en pleine évolution, et c'est pendant la rédaction de ce chapitre que la nature de la protéine en cause, la dystrophine, a été élucidée.

On peut s'attendre à d'importantes découvertes concernant la physiopathologie de l'affection, expliquant notamment son cours évolutif si particulier. En attendant d'éventuelles répercussions thérapeutiques, on peut espérer des progrès d'ordre diagnostique. Cependant la non expression très probable de la dystrophine dans le trophoblaste et les amniocytes empêche d'imaginer pour le moment un diagnostic prénatal phénotypique. Dans ce domaine et dans celui de la détection des conductrices la génétique moléculaire semble conserver tout son

\section{Summary}

The DMD gene, responsible for Duchenne and Becker muscular dystrophies, has been mapped to Xp21. DNA segments from this region were used as useful markers for prenatal diagnosis and carrier detection. Among them sequences that are highly conserved in different species were isolated and used to detect a large transcript expressed in squelettal muscle. The corresponding gene is huge, covering more than $2000 \mathrm{~kb}$. This unusual size is compatible with: (1) the observed high mutation rate; (2) the variable breakpoints observed in the $\mathrm{X}$; autosome translocations responsible for DMD in females; (3) the heterogeneity of deletion breakpoints; (4) the existence of intra-genic recombinations. By reading the nucleotide sequence of the coding regions, a new protein, dystrophin, was discovered. It is localized in triads, a muscular ultra-structure in which plasmic membrane comes into contact with sarcoplasmic reticulum. Its role - structural or functional - is not yet defined.

\section{Remerciements}

Les travaux personnels cités dans cet article ont bénéficié d'un financement de l'Inserm et de l'association française contre les myopathies (AFM). J.-C. Kaplan est boursier de l'AFM.

\section{TIRÉS A PART}

J. Chelly : Institut de pathologie moléculaire, Inserm U. 129, CHU Cochin, 24, rue du Faubourg-Saint-Jacques, 75014 Paris, France. 